Aus der chirurgischen Universitätsklinik von Professor $\mathrm{K} \mathrm{o} \mathrm{c} \mathrm{h} \mathrm{e} \mathrm{r}$ in Bern.

\title{
Akute hämorrhagische Pankreatitis durch Frühoperation geheilt.
}

\author{
Von Dr. Leopold Imfeld, I. Assistenzarzt der Klinik.
}

(Mit 2 Kurven.)

\section{K 1 in is che r Teil.}

Die akute hämorrhagische Pankreatitis gehört zu den seltenen Affektionen. D r e es m a n n hat in der Münchner med. Wochenschrift im April Igog eine Zusammenstellung dieser Krankheit publiziert. Er fand in der gesamten Weltliteratur II 8 operativ und 39 intern behandelte Fälle. Ebenso selten, wie die Krankheit als solche ist, ebenso selten wurde die richtige Diagnose gemacht. Meist brachte erst der Operations- oder Sektionstisch volle Klarheit über die Affektion. Gewöhnlich wurde wegen Ileus, Peritonitis, Appendicitis, Cholelithiasis operiert. Immerhin wurden mehrere Male richtige Wahrscheinlichkeitsdiagnosen gemacht, so von Körte, Mikulicz, Mayo Robson, $\mathrm{H}$ a h $\mathrm{n}$ und anderen.

Ich bemerke, daß im vorliegenden Falle, den Herr Professor $\mathrm{K}$ o c h e $\mathrm{r}$ im November 1908 operierte, schon vor der Operation die fast sichere Diagnose gemacht wurde.

Ich bringe nachfolgend die Krankengeschichte in Extenso, um so mehr, da einige interessante Abweichungen vom gewöhnlichen Verhalten sich zeigen.

A n a m n e s e v o m 23. XI. rgo8. F. H., 36 jähriger Mechaniker. Sonnabend, den I4. XI. erbrach Pat. zum ersten Male abends nach reichlichem Biergenusse. Hierauf schlief er gut. Sonntags, den I5. XI. trat mehrmaliges Erbrechen ein. Montag, den I6. XI. bis Mittwoch, den I8. XI. erbrach Pat. täglich einige Male, ganz besonders am Mitt- 
woch. Zugleich hatte er beständig Schmerzen im ganzen Abdomen, besonders aber in der Magengegend. Die Schmerzen waren kontinuierlich, hier und da mit größerer Kraft einsetzend, doch ging Pat. die ganze Woche noch der Arbeit nach. Über Stuhl und Winde weiß er nichts; nur erinnert er sich, am Freitag, den 20. XI. eine diarrhoische Stuhlentleerung gehabt zu haben.

Freitag, den 20. XI. und Sonnabend, den 2I. XI. waren die Schmerzen nur mehr ganz gering und kein Erbrechen mehr vorhanden. Sonnabend, den 2I. XI. nach Genuß von 5 mal 2 Deziliter Wein, erbrach Pat. neuerdings. Nach einer ruhigen Nacht bekam Pat. tags darauf, also Sonntag, den 22. XI. wieder Schmerzen im Abdomen und wieder besonders in der Magengegend. Nachmittags gleichen Tages erbrach Pat. ca. 20 Male. In der Nacht vom 22. auf den 23. schlief Pat. Heute, den 23. XI. morgens, hatte Pat. weniger Schmerzen und ging zur Arbeit. Um 1/2 Io Uhr ungefähr kamen aber so heftige Schmerzen, daß Pat. von der Arbeit weg nach Hause gehen mußte. Seither erbrach er bis abends 7 Uhr ca. 30 Male. Das Erbrochene bestand aus der jeweils aufgenommenen Nahrung: Milch und Tee und nachher aus Schleim. Hie und da war das Erbrochene leicht gallig. Häufig bestand schmerzhafter Singultus. Die Schmerzen sind beständig, sehr heftig und hie und da akut anschwellend. Seit Freitag kein Stuhl mehr. Heute I mal Winde.

Pat. hatte früher hie und da leichte Magenschmerzen, auch erbrach er oft, aber meistens nur, wenn er viel Bier getrunken hatte. Nie Ikterus.

Pat. war sonst stets gesund.

Der konsultierte Arzt sandte Pat. als Ileus hierher.

Eintrittsstatus vom 23. XI., abends 7 Uhr. Pat. ist sehr unruhig, liegt nur für einige Sekunden still da, dann schreit er laut auf und klagt über sehr heftige, kolikartige Schmerzen median am Nabel und hauptsächlich zwischen Nabel und Processus xyphoid. Beständig leidet er an starkem Singultus. Alle Io-I5 Minuten erbricht er einige Kubikzentimeter schleimiger Flüssigkeit, die keinen besonderen Geruch aufweist. Atmung wenig beschleunigt. Puls 48, leicht unregelmäßig, von eher geringer Spannung und guter Füllung. Temperatur 36,o. Pat. sieht blaß aus. Zunge feucht, mäßig belegt. Von Zeit zu Zeit macht Pat. mit dem Körper, namentlich Kopf und Rumpf, oszillierende Bewegungen wie einer, der Schüttelfrost hat. Abdomen bei Inspektion kaum aufgetrieben, keine umschriebene Vorwölbung, keine Darmzeichnung. Perkussion ergibt Dämpfung median über dem Nabel, sowie in dem größten Teil der rechten Bauchseite. Links tympanitischer Schall. Muskulatur im Epigastrium mäßig gespannt, im übrigen aber nicht. Peritoneale Reizung bei Druck am Nabel und oberhalb desselben. Blinddarmgegend sowie linke Bauchseite nicht druckempfindlich. Nirgends, abgesehen von der geringen 
Kontraktion der Recti oben, eine fühlbare Resistenz. Kein Tumor, keine Darmschlingen fühlbar. Kein freier Erguß, da links auch die abhängigen Partien tympanitisch schallen.

Urin: Etwas Eiweiß, kein Zucker, eine Spur Indikan, kein Gallenfarbstoff.

Wahrscheinlichkeitsdiagnose: Akute Pankreatitis.

Operationsbericht vom 23. XI. Igo8, abends 8 Uhr. Morph. o,or. Ruhige Bromäthyl-Äthernarkose. Schnitt median oberhalb des Nabels, I6 cm lang. Dicker Pannikulus subkutan sowie zwischen Fascie und Peritoneum. Eröffnung des Peritoneums. Kein Erguß. Fettreiches Netz. Serosa überall glatt und glänzend. Colon transv. klein. Einige Dünndarmschlingen sichtbar; sie sind klein und kontrahiert. Magen steht mit großer Kurvatur in Nabelhöhe. Nach Herausziehen des Magens wird oberhalb der kleinen Kurvatur das Pankreas nach Spaltung des kleinen Netzes fühlbar und sichtbar. Es fühlt sich groß, sehr derb an. Die Besichtigung ergibt, daß der größte Teil des Pankreas, mit Ausnahme der Kaputpartie prall hä mor$\mathrm{rhag}$ i sch, d u n elblau-schwärzlich ist. Lappenzeichnung weniger deutlich als sonst. Gallenblase und Gallenwege sind frei.

Diagnose: Akute hämorrhagische Pankreatitis.

Spaltung der Pankreaskapsel quer $3-4 \mathrm{~cm}$ lang. Es blutete anfangs wenig, bei tieferem Schneiden mehr. Tamponade nach M i k u li c $z$ mit Sack aus Jodoformgaze, der auf dem Pankreas aufliegt und mit mehreren Gazen gefüllt ist. Dazu ein dicker Glasdrain. Naht von Peritoneum und Fascie zu $3 / 4$ bis zu dem oben herausgeleiteten Tampon und Drain. Hautnaht mit Ausnahme von oben. Kollodialverband.

Puls nach der Operation etwas frequenter und unregelmäßiger. Füllung ziemlich gut. Spannung etwas gering. Pat. erhält sofort eine Kochsalzinfusion.

Der unmittelbare Erfolg der Operation war insofern ein sehr bemerkenswerter, als die heftigen Schmerzen fast vollkommen verschwanden, während das Erbrechen noch fortdauerte.

Fortsetzung der Krankengeschichte.

24. XI. Während der Nacht erbrach Pat. sehr oft schwarz-grüne Massen. Retentio urinae machte Katheterismus nötig. Abdomen wenig druckempfindlich. Leichter Ikterus der Konjunktiva. Puls 60. Häufiger Singultus. Tampon heraus.

Urin: Spur Eiweiß, kein Zucker, etwas Gallenfarbstoff, wenig Indikan, Urobilin negativ.

25. XI. Kein Erbrechen. Häufig Singultus. Wunde schön. Keine Winde trotz Darmrohr. Täglich 2 Infusionen. Per os etwas Tee. Urin wie am 24. XI. 
26. XI. Noch keine Winde. Abdomen leicht tympanitisch. Leberdämpfung verkleinert. Singultus.

Urin: Reichlich Zucker, Spuren Eiweiß, kein Indikan, kein Gallenfarbstoff, kein Urobilin.

27. XI. Nachts reichlich Windabgang. Abdomen weniger aufgetrieben. Selten Singultus. Puls gut.

Sahlis Glutoidreaktion nach 7 Stunden positiv.

Urin: Zucker positiv.

28. XI. Heute eine gut geformte und gefärbte Stuhlentleerung.

Urin: Zucker $1 / 4$ Proz. Noch Singultus.

29. XI. Noch Singultus.

Urin: Eiweiß negativ. Zucker $1 / 2$ Proz. (Die quantitative Zuckerbestimmung wurde nach der Pavyschen Titriermethode und mit dem Gärungssaccharometer gemacht.)

Indikan, Gallenfarbstoff und Urobilin negativ.

30. XI. Vormittags kein Singultus, nachmittags tritt er wieder auf. Abdomen und Wunde normales Verhalten. Auf beiden Lungen h. u. feuchte Ronchi. 2 mal tägl. I, 5 Natr. salicyl.

Urin: Kein Eiweiß, kein Urobilin, kein Indikan, kein Gallenfarbstoff. Zucker $1 / 8$ Proz.

I. XII. Kein Singultus. Natr. salicyl. 2 mal 1,5 .

Urin: Zucker schwach positiv.

2. XII. Urin zuckerfrei.

3. XII. Urin zuckerfrei.

5. XII. Urin zuckerfrei.

6. XII. Links h. u. über der Lunge starke Dämpfung bis zur 8. Rippe reichend. Abgeschwächtes Atmen und aufgehobener Pektoralfremitus daselbst. Probepunktion ergab ein sero-fibrinöses Exsudat. Kulturen blieben steril.

7. XII. Morgens Verband ganz durchfeuchtet von einer gelblichen, schleimigen, alkalischen Flüssigkeit. Die Untersuchung dieser Flüssigkeit ergab Pankreassaft und Eiter. Die genauere Analysierung folgt im 2. Teile der Arbeit. Das Sekret wurde nun lange Zeit hindurch mit der bekannten Kapsel aufgefangen, die F r e y e r für die Prostatektomierten angab, und in zwei sterile Flaschen abgeleitet. In der ersten Zeit betrug die Menge des Sekretes 200 und etwas mehr, bald nahm sie aber ab. Immerhin schloß sich die Fistel erst nach 4 Monaten ganz.

Sahlis Jodoform-Glutoidversuch wurde noch öfter wiederholt, immer mit dem nämlichen positiven Erfolge.

Die Stühle waren stets gut gefärbt.

Zucker wurde im Urin nicht mehr nachgewiesen.

22. XII. Heute ging durch die Fistel ein Stück sequestriertes Gewebe ab. Größe: I1/2: I : 1/2 cm. Mikroskop.: Zahlreiche Kern- 
trümmer, nekrotisches Fettgewebe und Hämorrhagien. Pankreasgewebe nicht erkennbar.

28. XII. Heute wurde ein ähnliches, etwas größeres Stück ausgestoßen wie am 22. XII. Es besteht mikroskopisch aus Bindegewebe. das größtenteils nekrotisch ist und sehr zahlreiche Fettröpfchen enthält. Hie und da finden sich auch Nekrosen des Fettgewebes mit zahlreichen Büscheln von Fettsäurekristallen. Pankreasgewebe nicht erkennbar. Sehr zahlreiche Häufchen von Kokken.

Puls blieb die ersten I2 Tage zwischen 60 und 70 , und ging nur 2 mal höher als 8o. Vom I2. Tage ab bewegte er sich zwischen 80 und 120. Temperatur stieg am 2. Tage nach der Operation auf 38,2 und blieb von da ab lange Zeit um 38 herum, nur 2 mal auf 39 und etwas mehr steigend.

Ät iologie.

Die Ätiologie der akuten hämorrhagischen Pankreatitis ist eine sehr mannigfache. Ich habe in der Literatur der letzten Jahre folgendes als Ursache angegeben gefunden:

Gallensteine, Magen- und Darmaffektionen, Mumps, Alkoholismus, Typhus, Appendicitis, Trauma, Embolie, Ulcus ventriculi, Ulcus duodeni, Malaria, Lungentuberkulose, Syphilis, Bronchitis, Adipositas, Oxalsäurevergiftung, Puerperium mit Blutverlusten, Arteriosklerose. - Auslösend wirkten oft: reichlich Alkohol, strenge Arbeit, kaltes Getränk, Traumen, der Defäkationsprozeß.

Von diesen zahlreichen Ursachen stehen die Gallensteine weitaus obenan, so sehr, daß Eg dahl in einer Zusammenstellung von I05 Fällen von akuter hämorrhagischer Pankreatitis 44 mal mit Sicherheit oder Wahrscheinlichkeit Gallensteine fand. Sehr häufig sind auch Magen- und Darmkatarrhe der Erkrankung vorangängig nachgewiesen. In der Zusammenstellung von E g d a h 133 mal. Auch unser Patient hat schon Tage vor der akuten Erkrankung an Erbrechen, Diarrhoe und Koliken gelitten. $\mathrm{Ob}$ diese Erscheinungen als Magen-Darmkatarrhe, bedingt durch das schwere Potatorium, anzusehen sind, oder ob sie bereits den Beginn der akuten hämorrhagischen Pankreatitis darstellen, ist nicht mit Sicherheit zu entscheiden. In der Reihe der Infektionskrankheiten steht Typhus und Mumps ätiologisch obenan. Ersterer wird im Sinne eines Darmkatarrhs wirken, für letzteren scheint das Pankreas eine Organdisposition aufzuweisen, ähnlich 
wie der Testis. In der Zusammenstellung von Eg dah 1 war II mal Mumps vorangängig und $\mathrm{Edgecombe}$ will bei einer Epidemie von Parotitis epidemica in einer Knabenschule von 33 Fällen $5 \mathrm{mal}$ eine leichtere metastatische Entzündung des Pankreas beobachtet haben. Klinisch äußerten sich diese Fälle durch Erbrechen, Schmerzen, Spannung der Bauchmuskeln besonders im Epigastrium und Verstopfung. In 4 Fällen bestand Fieber, in 2 Fällen eine deutliche Schwellung in der Pankreasgegend.

Was den Alkohol und die Adipositas als ätiologisches Moment anbetrifft, so ist der Zusammenhang mit der Pankreatitis wohl ein indirekter, indem Alkoholismus Adipositas und Darmkatarrhe im Gefolge haben und diese letzteren dann als eigentlich ursächliches Moment anzusehen sind.

Die anderen angeführten ätiologischen Faktoren haben infolge ihrer Seltenheit geringere Bedeutung.

Was nun die Endursache der akuten hämorrhagischen Pankreatitis betrifft, so ist sie in einer in der Drüse vor sich gehenden Aktivierung des in der Drüse in normalem Zustande inaktiven Pankreasfermentes zu suchen. Durch diese Aktivierung wird der Pankreassaft befähigt, Epithelien und Bindegewebe des Pankreas zu verdauen und zu Fettnekrosen in und um das Pankreas zu führen, wobei es zu ausgedehnten Hämorrhagien kommt. Als solche aktivierende Substanzen sind Galle, Bakterien, Darmschleimhautextrakt, Duodenalinhalt und Trypsin experimentell nachgewiesen, und zwar ist die Wirkung dieselbe, ob sie direkt in die Drüse oder die Drüsenausführgänge eingespritzt werden. Poly a hat darüber schöne Versuche an Hunden gemacht.

Diese Versuche werfen nun ein neues Licht auf die Entstehung der akuten hämorrhagischen Pankreatitis, wenigstens in bezug auf deren häufigste Ursachen: Gallensteine, Darmkatarrhe und Potatorium. Durch einen Gallen- oder Pankreasstein oder einen Schleimpfropf wird an der Vaterschen Papille der Gallenabfluß gehemmt, die Galle staut sich an und fließt, wenn der Sekretionsdruck genügend wird, durch den Ductus pankreat. gegen das Pankreas hin. Dadurch wird das Pankreas zymogen aktiviert, und dies übt alsdann seine verheerende Wirkung auf Eiweiß und Fettgewebe der Umgebung aus. Dabei 
spielen natürlich auch die Bakterien eine Rolle, die infolge der Stauung im Ductus pankreat. leicht vom Darm aus in die Drüse herauf, oder von dem infizierten Ductus choledochus herüberwachsen können.

\section{Symptomatologie.}

In der neuesten Literatur wird das Bild der akuten hämorrhagischen Pankreatitis folgendermaßen geschildert: Plötzlich eintretende, außerordentlich heftige Schmerzen von kolikartigem oder andauerndem. Charakter, die meist im Epigastrium empfunden werden. Die Schmerzen strahlen in die linke Seite oder die linke Unterbauchgegend aus, manchmal nach rechts in die entsprechenden Partien. Häufig besteht ausstrahlender Schmerz nach dem linken Schulterblatt oder zwischen die Schulterblätter. Intensivster Druckschmerz manchmal nicht in der Magen-, sondern in der Gallenblasengegend.

Charakteristisch soll der Mayo-Robsonsche Druckpunkt sein. Er liegt auf der Hälfte des Weges zwischen dem Ende der 9. Rippe und dem Nabel, und ferner ein Punkt über dem rechten Rectus abdominis und etwa Io $\mathrm{cm}$ oberhalb des Nabels, falls Ulcus duodeni ausgeschlossen werden kann. Ferner besteht häufig Übelkeit, Aufstoßen und Erbrechen. Letzteres meist gallig und oft sehr kopiös. Singultus kommt hie und da vor und ist dann nach D reesmann von übler Vorbedeutung. Stuhl- und Windverhaltung. Bauchdecken sind weich, leicht eindrückbar, keine Defense musculaire. Manchmal ist eine tiefliegende, sehr sehmerzhafte Resistenz im Epigastrium fühlbar. Hie und da Ptyalismus. Urin: Zucker meist negativ, hie und da Albumen. Der Indikangehalt ist nach D reesma n n für die Diagnose unwesentlich. Öfters gelingt der Nachweis von Gallenfarbstoff.

Neben diesen Symptomen bestehen schwere Allgemeinerscheinungen: Pat. kollabiert, Puls klein und frequent, Temperatur normal oder erhöht. Starke Unruhe. Hie und da Ikterus oder Odeme.

Das ist das Bild im Anfang der Erkrankung, bevor die Zeichen schwerer Fettgewebsnekrose und diffuser Peritonitis eingetreten sind. 
Wenn wir daraufhin die Symptome unseres Falles mit dem geschilderten Symptomenkomplex vergleichen, so finden wir Ubereinstimmung bis auf einige interessante Punkte:

I. Statt der in allen Lehrbüchern und. Publikationen beschriebenen Pulsbeschleunigung hatten wir in unserem Falle eine exquisite Pulsverlangsamung. Beim Eintritt betrug der Puls 48 und erreichte auch in den folgenden Tagen nur Werte zwischen 60 und 70 Pulsschlägen pro Minute. Ich habe das Vorkommen dieses Symptoms nirgends erwähnt gefunden in der gesamten Literatur.

Es dürfte sich dieses Symptom analog dem Goltzschen Klopfversuch erklären. Das geschwellte Pankreas drückt auf die Sympathicusganglien des Bauches, besonders auf den Plexus solaris. Dadurch wird dieser gereizt und der Reiz pflanzt sich fort auf die Medulla oblong. Hier springt er auf den Vagus über und Vagusreizung verursacht Herzverlangsamung. Vielleicht würde dieses Symptom öfters gefunden, wenn die Pat. in frühem Stadium der Erkrankung zur Beobachtung kämen, bevor an Stelle der Sympathicusreizung die Sympathicuslähmung getreten ist, durch die fortschreitende Schwellung des Pankreas und den Druck, den es ausübt.

2. Weitere interessante Einzelheiten bot der Urin, den ich täglich untersuchte:

a) Eiwei B. Albuminurie ist im allgemeinen selten. Albumen fand sich gleich beim Eintritt in geringer Menge und verschwand nach 3 Tagen gänzlich.

b) Z u cker. Zuckerausscheidung ist sehr selten. Beim Eintritt fand ich keinen Zucker. Am 3. Tage der Erkrankung schied Pat. plötzlich Zucker aus und dies dauerte 5 Tage. Die Menge des ausgeschiedenen Zuckers nahm langsam zu, stieg bis zu 1/2 Proz. und schwand dann langsam wieder. Die innere Sekretion des Pankreas erlitt also nur für kurze Zeit eine nachweisbare Schädigung, wohl durch eine vorübergehende entzündliche Schwellung und Läsion der Langerhansschen Zellinseln. Auch die äußere Sekretion der Drüse war nie so gestört, daß Ausfallserscheinungen eintraten. Es traten nie Fettstühle auf. Der Sahlische Jodoform- und Glutoidversuch war bei mehreren Versuchen immer und zu normaler Zeit positiv. 
Die Toleranz des Pankreas für Zucker war aber doch eine herabgesetzte. Größeren Anforderungen konnte das Pankreas nicht genügen, was folgender Versuch bewies, den ich am 14. Krankheitstage anstellte. Nach Einnahme von Ioo g Traubenzucker war der Urin des Pat. 24 Stunden lang zuckerhaltig. Die Menge des Zuckers stieg auf $1 / 2$ Proz.

c) Galle nfarbstoff. Beim Eintritt fehlte er. Am Tage nach der Operation war etwas Gallenfarbstoff nachweislich und auch leichter Ikterus conjunctivae. Am zweitfolgenden Tage war beides verschwunden. Es kam also nur zu einer vorübergehenden und leichten Gallenstauung infolge Kompression des Duct. choledoch. durch den geschwellten Pankreaskopf.

d) Indikan. Beim Eintritt war es nur spurweise vorhanden. D r e e s m a n n hält den Indikangehalt für die Diagnose belanglos. Mir scheint gerade der Indikangehalt ein Fingerzeig für die Diagnose zu geben, da er bei den bei der Differentialdiagnose am meisten in Betracht kommenden Affektionen: Ileus und Peritonitis vermehrt ist.

e) Urobilin fand sich nie.

3. Darf erwähnt werden, daß trotz des hartnäckigen Singultus mit seiner ominösen Vorbedeutung der Pat. am Leben blieb.

\section{Therapie.}

Die Therapie kann nur eine chirurgische sein, und zwar soll auch schon bei Verdacht auf akute hämorrhagische Pankreatitis zur Laparotomie geschritten werden. Von 39 intern behandelten Fällen starben nach der Zusammenstellung von D rees man $\mathrm{n}$ 36, 3 genasen, was einer Mortalität von 93 Proz. entspricht. Die drei geheilten Fälle wurden von Brentano, Z eller und $\mathrm{J}$ a k o b beschrieben.

Die chirurgische Therapie besteht in der Freilegung des Pankreas durch Spaltung des kleinen Netzes oder des Lig. gastrocolicum, Ausspülen einer event. vorhandenen Jauchehöhle mit sterilem Wasser, Incision des Pankreas, Tamponade, event. Drain. Es ist dies ein Verfahren, wie es auch in unserem Falle von Herrn Prof. K o c h e r eingehalten wurde.

Uber den Wert der operativen Hilfe geben folgende Zahlen Aufschluß: von iı 8 operativ behandelten Fällen, die Drees- 
$\mathrm{m}$ a $\mathrm{n} \mathrm{n}$ aus der Literatur zusammenstellte, starben $6 \mathrm{r}$, was einer Mortalität von 55 Proz. entspricht. Von diesen II8 Fällen waren aber nur 40 mit Tamponade des Pankreas behandelt worden, und von diesen 40 starben nur 8, was 20 Proz. Mortalität ausmacht. Noch einen weiteren Fortschritt in der Therapie bedeutet die Incision des Pankreas. Nach $\mathrm{H}$ ah $\mathrm{n}$ s Vorschlag sprechen Mikulicz und in neuerer Zeit Bornhaupt ihr energisch das Wort. - Neuerdings berichtet Fly $\mathrm{n} n$ über einen Fall, den er ohne Tamponade und Drainage behandelte und der trotzdem am Leben blieb.

Um die zurückbleibende Pankreasfistel zu schließen, wird die Wohlgemuthsche Diät, bestehend in Vermeidung von Kohlehydraten und Zufuhr von Nat. bicarb. warm empfohlen.

Auch wir empfehlen sie unseren Patienten. Pat. konnte sie aber aus finanziellen Gründen nicht strikte durchführen. Die Fistel schloß sich in unserem Falle nach ca. 4 Monaten.

\section{Physiologischer Teil.}

Die Untersuchungen des menschlichen Pankreassaftes sind nicht sehr zahireiche. Es beziehen sich die Untersuchungen meistens auf die Beschaffenheit des Inhaltes von Pankreascysten und des Sekretes von Fisteln, die nach Pankreascystenoperationen zurückblieben. Es handelt sich also meistens um mehr oder weniger pathologisches Sekret. Das erste vollständig normale menschliche Pankreassekret untersucht $\mathrm{zu}$ haben, ist das Verdienst von Dr. Karl Glaessner, der in einer vorzüglichen Arbeit ,Über menschliches Pankreassekret" die Ergebnisse seiner physiologischen Untersuchungen niedergelegt und in Hoppe-Seylers Zeitschrift für physiolog. Chemie im Jahre Ig04 veröffentlicht hat. Eine ebenfalls überzeugende Arbeit findet sich in der gleichen Zeitschrift von $\mathrm{O}$. S c h u $\mathrm{m}$ m unter dem Titel ,Uber menschliches Pankreassekret" vom Jahre I902. Doch handelt es sich hier nicht um vollkommen physiologische Verhältnisse, sondern um die Untersuchungen des Sekretes einer Pankreascyste und der sich daran anschließenden Pankreasfistel. Glaessner hatte die überaus seltene Gelegenheit, bei einer von $\mathrm{K}$ ör t e wegen Narbenstenose des Ductus choledochus operierten Patientin sämtliches Sekret 
des Pankreas aufzufangen und zu untersuchen. Ein feines Drainrohr, in den Duct. pankreat. eingeführt, leitete das Sekret 6 Tage lang nach außen.

Das Pankreassekret in unserem Falle ist auch keineswegs physiologisch, weil aus einem kranken Pankreas hervorgegangen und mit Eiter untermischt. Trotzdem wollte ich es einer genaueren experimentellen Untersuchung unterziehen, weil bei einer Materie, die so selten Gegenstand der Untersuchung wird, jeder Beitrag von Wert ist.

Ich vergleiche im folgenden die Resultate meiner Untersuchungen hauptsächlich mit den Untersuchungen von Glaessner, weil es sich hier um rein physiologische Verhältnisse handelt.

Menge und Beschaffenheit des Pankreassaftes.

Die tägliche Menge des Pankreassaftes schwankte in dem erwähnten Falle von Glaessner zwischen 420 und $848 \mathrm{ccm}$. In unserem Falle betrug das Maximum $250 \mathrm{ccm}$ und nahm alsdann $a b$ bis zum Schlusse der Fistel. Ein Teil davon kommt zudem auf Rechnung der Leukocyten, Fibrin und Delritus. Der bedeutende Unterschied der Quantität ist leicht durch die Annahme erklärlich, daß eben nur ein Teil des Pankreassaftes, und zwar der kleinere, durch die Fistel abfloß, der größere aber seinen normalen Weg durch den Ductus pankreat. in den Darm fand. Daß letzteres zutrifft, beweist uns die Untersuchung des Stuhles und der positive Ausfall des Sahlischen Jodofrom-Glutoidversuches (Seite I29).

Unser Pankreassaft war stark getrübt infolge der Entzündungsprodukte, wurde aber nach Zentrifugieren klar und gelblich. Reaktion war alkalisch, manchmal schwach sauer. Diese leicht sauere Reaktion wird auf Bakterienwirkung zurückzuführen sein, vielleicht auch auf Nukleinsäure, die sich durch Leukocytenzerfall bildete. Mikroskopisch fanden sich zahlreiche polynukleäre Leukocyten, Zelltrümmer, Delritus. Bakterien fehlten. Kulturell wurden Staphylokokken nachgewiesen, die vielleicht nur durch Herunterwachsen vom Wundrande ins Sekret gelangten. 
Die analytische Untersuchung unseres Pankreassaftes, ausgeführt von Herrn Dr. $\mathrm{Z}$ a t e c k i im hiesigen physiolog.-chem. Institut, ergab folgende Zusammensetzung, die ich in Vergleich stelle zu den analytischen Untersuchungen einer Portion a des Falles G $\mathrm{l}$ a ess ner und den Untersuchungen einer Portion B der Pankreasfistel des Falles $\mathrm{Schu} \mathrm{m} \mathrm{m}$. Ich betone noch, daß ich, um den Saft möglichst von Eiter zu befreien, wie zu allen meinen Untersuchungen auch zu dieser Analyse den zentrifugierten Saft verwendete. War der Saft schwach sauer, so machte ich ihn durch Zusatz von einigen Tropfen $1 / 10$-Normallauge schwach alkalisch.

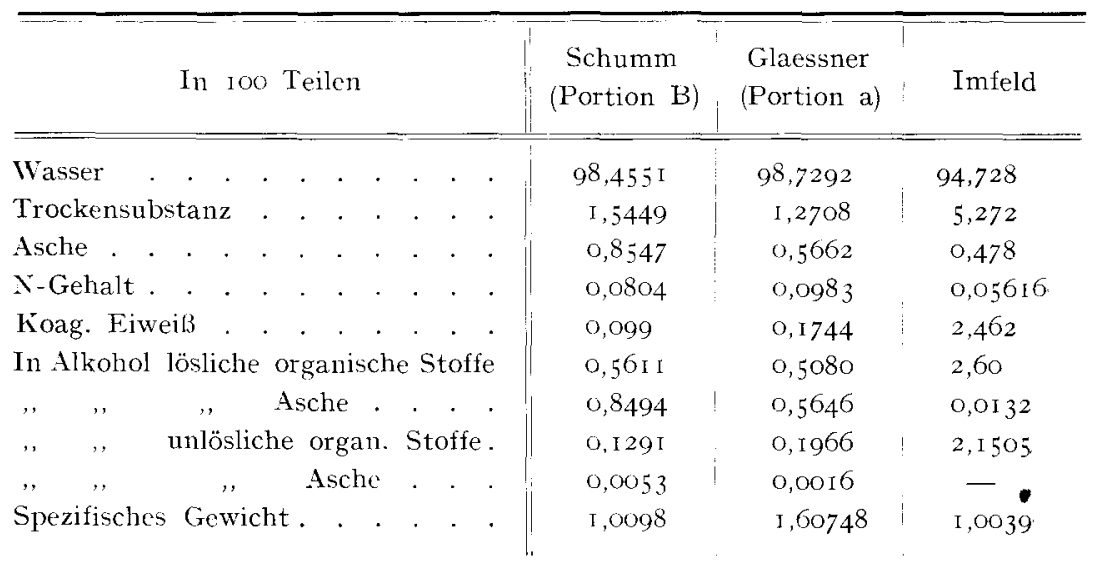

Die Unterschiede in der Analyse werden wohl durch die Beimengung der Entzündungsprodukte, die sich natürlich durch die Zentrifuge nicht ganz entfernen lassen, zu erklären sein.

Untersuchungen über die Fermente.

I. Trypsin. Daß der Pankreassaft an und für sich keine tryptischen Eigenschaften hat, ist eine sichere Tatsache der Physiologie. Das Trypsin ist im Pankreas in Form eines Profermentes vorhanden. Es ist inaktiv und bedarf der Aktivierung, um zur Wirkung zu gelangen. Auf dieses aktivierende Agens trifft der Pankreassaft, sobald er in den Darm eintritt. Es wird von $\mathrm{Paw}$ low Enterokinase genannt und ist ein Sekretionsprodukt der Darmschleimhaut. 
Ich untersuchte nun, wie unser Pankreassaft sich bezüglich der proteolytischen Wirkung verhält.

Ich machte zuerst die Untersuchung mit dem leicht verdaulichen Karminfibrin. Einige Kubikzentimeter Saft wurden mit einer Flocke Karminfibrin in den Brutschrank gestellt. Nach 24 Stunden war die Flocke verdaut und die Flüssigkeit leicht rötlich gefärbt. Es schien also dieser Saft in der Tat proteolytische Wirkung zu haben. Um diese genauer $\mathrm{zu}$ studieren, machte ich folgende Versuchsreihe:

Probe I. Einige Kubikzentimeter Pankreassaft u. Karminfibrin,

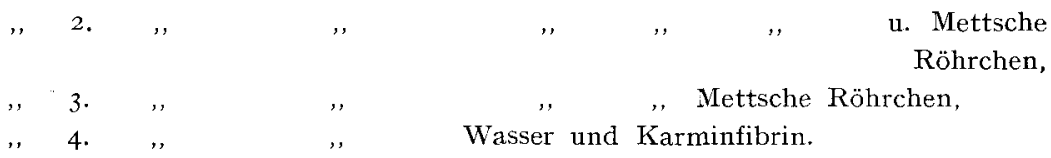

Diese Proben wurden 24 Stunden im Brutschrank gelassen, alsdann zeigte sich:

in Probe I Fibrin verdaut,

, , 2 ,, ,, Mettsche Röhrchen intakt,

, , , 3 Mettsche Röhrchen intakt,

, " 4 Fibrin unverdaut.

Aus dieser Versuchsreihe zog ich folgende Schlüsse: Das Karminfibrin scheint eine Enterokinase in sich zu haben (Probe I), da és vom Pankreassaft verdaut wird, welcher das Hühnerejweiß in den Mettschen Röhrchen intakt läßt (Probe 3); hingegen scheint es nur jene Teile des Pankreassaftes zu aktivieren, die mit ihm direkt in Berührung kommen und nur soviel, wie zur eigenen Verdauung genügt, da bei Probe 2 die Mettschen Röhrchen intakt bleiben. (Wahrscheinlich ist es der $\mathrm{K}$ a $\mathrm{lk}$ gehalt des Fibrins, der das Trypsin aktiviert hat. Nach den Untersuchungen von Delezenne aktivieren Kalksalze ähnlich wie Enterokinase.) Probe 4 zeigt, daß das Karminfibrin keine Selbstverdauung aufwies.

Bei dem eigentümlichen Verhalten des Karminfibrins dachte ich auch daran, ob nicht event. Bakterien die Aktivierung besorgen, doch schien mir dies ausgeschlossen, da Probe 3 keine Verdauung aufwies.

Ich suchte nun den Pankreassaft zu aktivieren. Herr Professor Kocher hatte die Freundlichkeit, mir etwas frischen 
menschlichen Darmsaft zur Verfügung zu stellen, den er bei einer Gastroenterostomie dem Darme entnahm. Später habe ich die Versuche mit Darmpreßsaft, der mit der Buchnerschen Presse hergestellt wurde, wiederholt. Es zeigte sich, daß unser Saft proteolytische Wirkung aufwies, aber in geringem Maße. Ich lasse die Versuchsreihe folgen:

Probe I. Eine kleine Schnitte Hühnereiweiß (ro mg) u. Pankreassaft u. Darmsaft,

, 2. "Mettsche Röhrchen

"4. ",

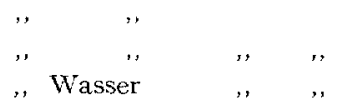

Nach 24 Stunden Brutschranktemperatur war Probe $\mathrm{I}$ fast verdaut, Probe 2 unverdaut, bei Probe 3 jederseits I mm verdaut, Probe 4 unverdaut, d. h.: Der Pankreassaft wurde durch den Darmsaft aktiviert (Probe I und 3). Daß sowohl der Pankreas- wie der Darmsaft allein nicht verdauten, beweisen die Proben 2 und 4.

2. Li pase. Zur Untersuchung auf die fettspaltende Wirkung des Pankreassaftes nahm ich steriles Olivenöl, das zuerst auf event. schon vorhandene Fettsäuren genau geprüft wurde. Ich lie $B$ je I ccm Pankreassaft auf Io ccm Olivenöl eine bestimmte Zeit einwirken. Alsdann titrierte ich mit $1 / 10^{-N o r m a l l a u g e ~ n a c h ~}$ Zusatz von etwas Alkohol und unter Verwendung des Phenolphthaleins als Indikator. Die Menge der zur Titration nötigen Kubikzentimeter $1 / 10^{-N o r m a l l a u g e}$ gibt einen Begriff von der Menge der gebildeten Fettsäuren. Folgende Tabelle gibt die gefundenen Werte wieder:

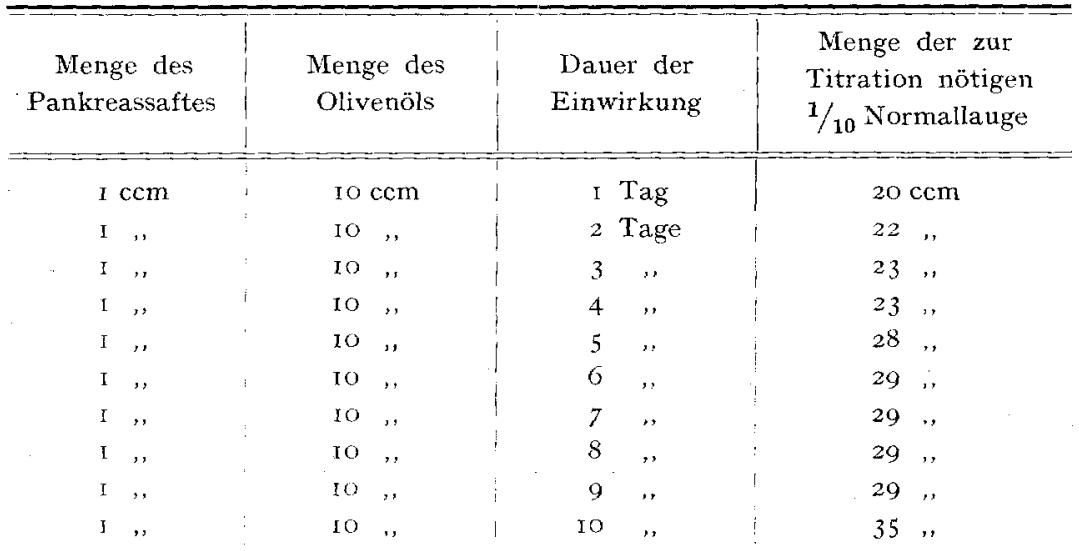


Die fettspaltende Wirkung war demgemäß eine ziemlich erhebliche und es bedurfte dazu keineswegs eines aktivierenden Agens. Woher die starke Steigerung der Fettspaltung am ro. Tage kommt, ist mir nicht erklärlich.

3. Diastase. Die Untersuchung auf das diastatische Ferment wurde durch Einwirkung des Saftes auf Stärke gemacht. Ich löste 6,0 Amylum in 200,0 Wasser und setzte 20,0 des Pankreassekretes bei. Ich untersuchte nun an den folgenden Tagen auf Zucker mittels der Trommerschen Probe. Zur quantitativen Zuckerbestimmung benutzte ich die Pavysche Titriermethode. Es ergab sich:

I. Nach 24 Stunden 1/2 Proz. Zucker-Jodreaktion auf Amylum positiv.

2. ", 2 Tagen

3. , 3

4 .

Nach 2 Tagen war also das Maximum der Diastase vorhanden. Auch nach 4 Tagen war noch Amylum da.

Anders gestaltete sich die Diastase, wenn statt der rohen gekochte Stärke genommen wurde in den gleichen Mengenverhältnissen und der gleichen Einwirkungszeit wie oben. Sofort nach Zusammenbringen von Stärke und Saft war die Trommersche Probe positiv. Nach 24 Stunden der Einwirkung war die Jodreaktion auf Amylum negativ. Die Menge des Zuckers war nach 24 Stunden $x$ Proz. und steigerte sich in den folgenden Tagen um nichts mehr.

4. La b f erment. Es ließ sich nicht nachweisen.

Untersuchungen über die Menge und dieverdauende Kraft des in den einzelnen Stunden nach einer Probemahlzeit sezernierten Saftes.

Um ein Bild zu bekommen von dem Einflusse der Nahrung auf die Menge des sezernierten Saftes wurde dem Patienten eine Probemahlzeit gegeben, bestehend aus $40 \mathrm{~g}$ Fleisch, I2 $\mathrm{g}$ Butter und $70 \mathrm{~g}$ Kartoffel. Nachher wurde der Saft stündlich extra aufgefangen. Nüchtern flossen $4-8 \mathrm{ccm}$ pro Stunde. Den Einfluß der Nahrung auf die Saftmenge ergibt folgende Kurve (Siehe Fig. I). 
Der erste Anstieg beruht auf dem Reiz der Salzsäure des Magensaftes, der Anstieg in der zweiten Stunde nach dem Übertreten der Ingesta in den Darm. Im Falle von Glaessner trat das Maximum der Absonderung erst in der 4. Stunde auf. Die Erklärung für das abweichende Verhalten in diesem Falle liegt darin, da $B$ Patient eine ausgezeichnete Magenmobilität hat, wie eine vorgenommene Magenuntersuchung mit Hilfe des Ewaldschen Probefrühstückes ergab. Damit in Verbindung steht auch der rasche Abfall der Sekretion, die schon nach der 5. Stunde zur Norm zurückkehrt.

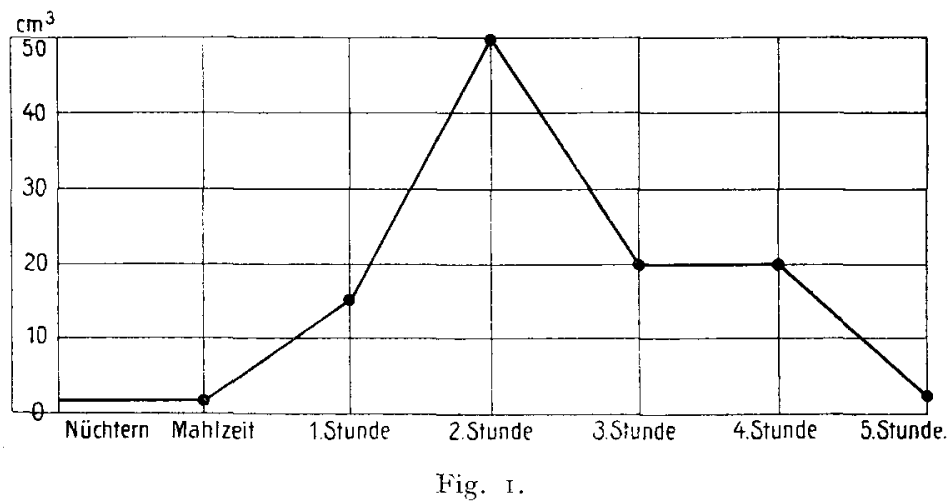

Die 4 Portionen des stündlich aufgefangenen Saftes wurden hierauf auf ihre verdauende Kraft getrennt untersucht, um zu eruieren, wie sich das Verdauungsvermögen des Pankreas in den einzelnen Stunden der Verdauung verhält.

I. Untersuchung der Lipase.

Io ccm Olivenöl wurden mit I ccm des Sekretes der I, 2., 3. und 4. Stunde 24 Stunden lang in den Brutschrank gestellt und entsprechend numeriert. Zur Neutralisation der gebildeten Säure war alsdann nötig:

$$
\begin{aligned}
& \text { bei Probe } I=7 \quad \mathrm{~cm} \mathrm{1/10} \text {-Lauge } \\
& \text {, , } 2=7,5 \text {,, ,, } \\
& \text {,, , } 3=9 \quad,, \quad \text {,, } \\
& \text { ", } 4=7 \quad,,
\end{aligned}
$$

Das Maximum der fettspaltenden Kraft fällt also in unserem Falle in die 3. Stunde, während sie bei Glaessner in die 
4. Stunde fällt, ein Unterschied, der sich leicht erklärt durch die erwähnte starke Motilität des Magens und den schnellen Abschluß der Verdauung.

2. Untersuchung der Diastase.

Ich ließ zu dem Zwecke je I ccm Saft von jeder der 4 Portionen auf $20 \mathrm{ccm} 3 \mathrm{proz}$. ungekochter Stärke wirken. Nach 24 Stunden ergab die Titration:

$$
\begin{gathered}
\text { bei Probe I }=0,4 \% \text { Zucker } \\
\text {, " } \quad 2=0,25 \% \quad, \\
, \quad, \quad 3=0,33 \% \quad, \\
, \quad, \quad 4=0,3 \% \quad,
\end{gathered}
$$

Dieses Resultat steht ziemlich in Übereinstimmung mit den Glaessnerschen Untersuchungen, mit dem Abfall der diastatischen Kraft in der 2. und 4. Stunde; nur ist bei Gla essner die diastatische Kraft entgegen unseren Untersuchungen in der 3. Stunde etwas größer als in der I.

3. Untersuchung der tryptischen Wirkung.

Die Untersuchung der tryptischen Wirkung der 4 Proben mit Hilfe der Mettschen Röhrchen unter Hinzufügung von Darmpreßsaft ergab keinen deutlichen Unterschied der 4 Proben, und war überhaupt gering.

Es wird auffallen, daß die Verdauungswirkung in dieser Versuchsreihe bedeutend geringer war als in den Versuchen, die ich anstellte zur „Untersuchung über die Fermente" im allgemeinen. Wir sehen dies besonders deutlich am fettspaltenden Ferment. Dort brauchte ich bei der gleichen Menge Olivenöl und Pankreassaft und der gleichen Digestionszeit $20 \mathrm{ccm}$ 1/10-Lauge zur Neutralisation der gebildeten Säure, hier nur $7 \mathrm{ccm}$. Auch die diastatische Wirkung erscheint etwas kleiner. Eine unzweideutige Erklärung für diese auffällige Erscheinung steht mir nicht zu Gebote; doch könnte event. folgendes eine Erklärung bieten: Die letzteren Untersuchungen wurden ungefähr I Monat nach den ersteren gemacht, also zu einer Zeit, wo bereits wenig Sekret geliefert wurde und die Krankheit sehr ins chronische Stadium gekommen war. Es ist nun wohl denkbar, daß zu dieser 
Zeit diejenigen Teile der Drüse, die ihr Sekret nach außen lieferten, durch chronisch-entzündliche Veränderungen in einen Zustand gekommen waren, in dem sie ein schwächeres, minderwertiges Sekret mit geringerer fermentativer Kraft lieferten.

EinfluB der Säuren und Alkalien a uf die Sekretionsmenge.

$\mathrm{P}$ a w low und seine Schüler haben nachgewiesen, daß die Pankreassekretion sich vermehrt, wenn Säuren auf die Schleimhaut des Duodenums einwirken. Ich lie $B$ bei unserem Patienten, der morgens früh eine Tasse Kaffee getrunken hatte, von 8-I2 Uhr alle halbe Stunden das Sekret extra auffangen. Um 9 Uhr gab ich dem Patienten 250,0 0,3 proz. HCl-Lösung zu trinken. Die Sekretmenge in den einzelnen halben Stunden war folgende:

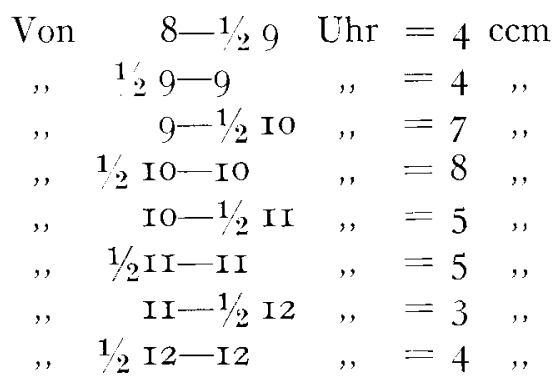

Man ersieht aus der Tabelle, daß die Wirkung der Säure eine sofortige ist und sich in der zweiten halben Stunde zum Maximum steigert, um dann sofort wieder zu sinken.

Bei einem 2. Versuche wurden $250 \mathrm{ccm}$ 0,5 proz. HCl-Lösung gegeben. In der Stunde vor der Einnahme flossen $9 \mathrm{ccm}$, in der Stunde nach der Einnahme $16 \mathrm{ccm}$, in der zweitfolgenden nur mehr 9 , und in der drittfolgenden 7 .

Was die Wirkung der Alkalisalze auf die Menge des Pankreassaftses betrifft, so sind die Autoren darüber uneinig. Während B e cker bei Hunden eine Herabsetzung der Sekretion beobachtete, fand Gla es s n e r bei seiner Versuchsperson gar keinen Einfluß auf die Saftmenge. Ich stellte den Versuch folgendermaßen an: Ich gab dem Patienten die gleiche Probemahlzeit, wie ich es in dem Kapitel ,Untersuchungen über die Menge und 
die verdauende Kraft des in den einzelnen Stunden nach einer Probemahlzeit sezernierten Saftes" angegeben habe, und gab nun dem Patienten zu Anfang der 2. Stunde, in der nach der in jenem Kapitel gezeichneten Kurve die größte Saftmenge zu erwarten war, $7 \mathrm{~g}$ Nat. bicarb. Folgende Kurve veranschaulicht das Ergebnis:

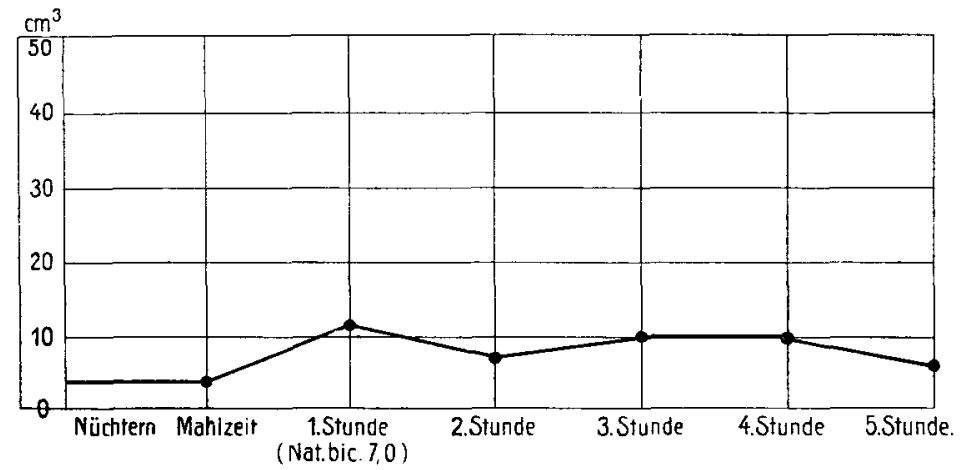

Fig. 2.

Wenn wir die beiden Kurven vergleichen, sehen wir, da $\$$ in der 2. Stunde statt des nach der früheren Kurve zu erwartenden Anstieges ein Abfall eintritt, und daß auch in der 3. und 4. Stunde die Sekretion eine geringe bleibt. Nach diesem Versuche ist also den Alkalisalzen eine exquisit sekretionshemmende Wirkung auf das Pankreas entschieden zuzuerkennen.

Anpassungsfähigkeit des Pankreas an die an dasselbe gestellten. Forderungen.

Man glaubte früher bewiesen zu haben, daß der Pankreasdrüse in hohem Maße eine Anpassungsfähigkeit an an sie gestellte Forderungen eigne in der Weise, daß jeder Nahrungsstoff zwar die Produktion aller 3 Fermente anrege, daß jedoch das Ferment in größtem Maße vorhanden sei, das ihn spalte. Bei Fettnahrung z. B. sollte das fettspaltende Ferment sehr stark sein und die beiden anderen Fermente, obwohl auch vorhanden, bei weitem überwiegen. Das gleiche schien für das diastatische und tryptische Ferment bewiesen bei Zufuhr von Kohlehydraten bzw. Eiweiß. 
Ich habe diesbezüglich auch Versuche angestellt, indem ich dem Patienten an drei verschiedenen Tagen einmal ein Beefsteak von $200 \mathrm{~g}$, das zweitemal Rahm $200 \mathrm{~g}$, das drittemal Kartoffel 200,0 verabreichte, und das daraufhin abgeschiedene Sekret auf die Stärke des betreffenden Fermentes untersuchte. Die Versuche mit dem Pankreassaft wurden gleich gemacht, wie sie im Kapitel „Untersuchungen über die Fermente" angegeben sind. Folgendes sind die Resultate:

I. Für das tryptische Ferment konnte ich keinen Unterschied finden.

2. Das fettspaltende Ferment war entschieden nach GenuB des Rahmes vermehrt gegenüber dem Fettspaltungsvermögen eines Durchschnittssaftes. Eine Anpassungsfähigkeit der Drüse schien somit zu bestehen. Ich bekam Werte, die zwischen 32 und $44 \mathrm{ccm} 1 / 10^{-L a u g e}$ schwankten, während beim Durchschnittssaft die Werte zwischen 20 und $35 \mathrm{ccm}$ sich bewegten. Ich betone noch, daB die beiden Untersuchungsreihen zu gleicher Zeit der Erkrankung gemacht wurden.

3. Auch das diastatische Ferment wies eine Vermehrung auf, indem schon nach 24 Stunden I 1/2 Proz. Zucker vorhanden war, der am 2. Tage auf 2 Proz. stieg und daselbst verblieb.

Nach B a b kin nun gibt es eine solche Spezialisierung nicht. Die 3 Fermente werden nach ihm parallel abgeschieden bei den verschiedenen Nahrungsmitteln. Bei Einnahme von Milch z. B. ist zwar das tryptische Ferment sehr gesteigert, allein in gleichem Maße ist auch das diastatische und fettspaltende Ferment vermehrt.

Es hätte mich nun interessiert, nachzuuntersuchen, ob bei Einnahme von Rahm z. B, wo ich das fettspaltende Ferment stark vermehrt fand, auch die beiden anderen Fermente eine entsprechende Vermehrung zeigen oder nicht, mit anderen Worten, ob eine Anpassungsfähigkeit der Drüse besteht in dem Sinne, daß immer das benötigte Ferment über die anderen überwiegt, oder ob B a bkin recht hat, der diese Spezialisierung nicht zugibt. In ersterem Falle hätte ich für das Trypsin und die Diastase kleine Werte finden müssen, in letzterem Falle große. 
Leider bin ich diese von $\mathrm{Babkin}$ aufgestellte Lehre zu spät inne geworden, zu einer Zeit, wo meine Pankreasquelle versiegt war.

\section{Literaturverzeichnis.}

I. Bergmann u. Bruns. Handb. d. prakt. Chir. 1907. 3. Aufl.

2. B r e n t a n o, Pankreasnekrose. Langenbecks Archiv, Bd. 6I, H. 4 .

3. H a h n. Deutsche Zeitschr. f. Chir, Bd. 58.

4. v. Mikulicz. Chirurgie des Pankreas. Grenzgeb. Bd. I 2.

5. Stockton and Willi a m s, Acute Pankreatitis. Buffalo med. journ. I908, Juli.

6. M a $\mathrm{g} \mathrm{m}$ a $\mathrm{n}$, Ein Fall von a.h. Pankr. bei Cholelith. Med. Korrespondenzbl. d. württemberg. ärztl. Landesvereins I908.

7. Edge combe, Metastatic affection of the pancreas in mumps. Practitioner Igo8, Februar.

8. Eng e $1 \mathrm{~m}$ a $\mathrm{n}$, Freie Vereinigung d. Chirurgen Berlins, 165. Sitzung am Io. VII. 1909. Zentralbl. f. Chir. I909, Nr. 40.

9. E g d a h 1, A review of hundred and five reported cases of acute Pankreatitis with special reference to etiology; with report of two cases. Johns Hopkins hospital bulletin I907, IV.

Io. E b n e r, Pankreatitis u. Cholelithiasis. Sammlung klin. Vorträge, Nr. 452/53. Leipzig, Breitkopf u. Härtel.

II. M a yo, The surgical treatment of pancreatitis. Surgery, gynecology and obstetrics, Vol. VII, No. 6. Zentralbl. f. Chir. Igog, Nr. I7.

I2. II a g g a r d, The etiology and pathogenesis of pancreatitis. Surgery, gynecology and obstitrics, Vol. VII, No. 6. Zentralbl. f. Chir. I909, Nr. I7.

13. Ochsner, The diagnosis of pancreatitis. Surgery, gynecology and obstetrics, Vol VII No. 6. Zentralbl, f. Chir. I909, Nr. I7.

14. Croftan, Pancreatic diabetes and its relation to gallstons. Surgery gynecology and obstetrics, Vol. VII, No. 6. Zentralbl. f. Chir. I909, Nr. I7.

15. Schröder, Physiology an chemical pathology of the pancreas in pancreatitis. Surgery, gynecology and obstetrics, Vol. VII, No. 6. Zentralbl. f. Chir. I909, Nr. I7.

16. Bor nha pt, Zur Kasuistik d. sogenannten akut. Pankr. Langenbecks Archiv, Bd. 32, H. 1 .

I7. Poly a, Zur Pathogenese d. akuten Pankreasblutung und Pankreasnekrose. Ber. klin. Wochenschr. Igo6, Nr. 49.

I8. Dreifu B, Beiträge zur Pankreaschirurgie. Deutsche Zeitschr. f. Chir. I 908, Bd. 43, H. 4 u. 5 .

19. Mayo-Robson, Beziehungen d. Anatomie zu den Krankheiten des Pankreas. Berl. klin. Wochenschr. 1908.

20. Dreesma n n, Diagnose u. Behandlung der Pankreatitis. Münchn. med. Wochenschr., April r9o9, Nr. I4. 
2I. If y n n, Report of a case of acute pankreatitis. Albany med. annals igog, April.

22. Truhart, Über die akuten Erkrankungsformen der Bauchspeicheldrüse. Petersburg. med. Wochenschr. Igo9, Nr. 7; Zentralbl. f. innere Medizin I $909, \mathrm{Nr}, 25$.

23. Gla essner, Über menschliches Pankrcassekret. Hoppe-Seylers Zeitschr. f. physiol. Chemie 1903/04, Bd. 40 .

24. S ch u m m, Über menschliches Pankreassekret. Ebenda 1902, Bd. 36 .

25. Deleze n ne, Über Enterokinase. C. R. Soc. Biol. 53-55.

26. A b derhalden, Lehrbuch der physiolog. Chemie. 2. Aufl. I909.

27. Nagel. Handbuch der Physiologie des Menschen. 1907.

28. Babkin, Zur Frage der sekretor. Tätigkeit der Bauchspeicheldrüse. Berichte der kaiserl. militärärztl. Akademie zu St. Petersburg, Jahrg. 9, Nr. 2 u. 3, S. 93, 1904. 